\title{
The Application of System identification method to characterize the performance of NiMH batteries in hybrid vehicles
}

\author{
Karin Kandananond \\ Valaya Alongkorn Rajabhat University, Department of Industrial Management, 13180 Prathumthani, Thailand
}

\begin{abstract}
Nickel-metal hydride (Ni-MH) battery is one of the electric sources which is widely used in hybrid electric vehicles. As a result, it is important to understand the characteristics of Ni-MH battery which is connected to direct current machine in the vehicle. However, the crucial problem is the complexity of the vehicle system which deals with the charging and discharging process of battery in order to maintain the designated speed. The system is considered as a black box and the system identification method is utilized in to characterize the dynamic behavior of the system. The system inputs are battery voltage, armature current and state of charge (SOC) while the output is the speed of DC machine. However, the system identification method will not work properly if the available data available has played an importable role on the determination of the model. As a result, the data regarding all parameters were collected and transmitted to the data logger and used to construct different models. The results from the system identification method indicate that the autoregressive model with exogenous input (ARX) is the most appropriate model to explain the relationship between inputs and output. Therefore, the performance of hybrid vehicle related to the characteristics of Ni-MH batteries is elaborately characterized and this study leads to the effective maneuver.
\end{abstract}

\section{Submitting the manuscript}

The characteristics of batteries are the critical factors affecting the performance of the electric vehicle which is driven by the direct current (DC) motor. DC motor is the main drive mechanism of the electric vehicle. Basically, the speed and torque of DC motor are adjusted by varying the amount of supply voltage and input current at the armature component. If the armature is supplied with the line voltage, the capability to control the speed of the motor is accurate and efficient since the value of line voltage is stable over the time and is considered as the infinite source. However, electric vehicles mainly rely on the power from batteries so the supplied voltage that DC motor receives is different than the one from line voltage. This is because the battery pack is the limited source of power supply because, at the supplying stage, they discharge the current to the sink. On the other hand, they are required to be charged in order to maintain the capability of supplying power. As a result, there is another factor which plays an important role on the supply of power to the motor, i.e., state of charge (SOC) or the gauge which measures the amount of battery charge. Moreover, since the charging characteristic of battery packs depends on the chemical reaction of different compounds, the study also focuses on one type of batteries, Nickel-metal hydride (NiMH). For Lithium-ion batteries, they work on the basis that Lithium-ion moves from one electrode to another. The advantages of Lithium-ion batteries are small memory effect, low self-discharge, and high energy density. For this reason, they are popular and commonly used for portable electronics. However, NiMH is based on the chemical reaction of the positive electrode (Nickel Oxide Hydroxide: $\mathrm{NiOH}$ ) and a negative electrode (alloy). Because of its energy density equivalent to the one of Li-ion, it is also widely used in the hybrid cars and portable electronic devices. The spotlight of this study has two folds: one is the characteristic study of these two types of batteries. Another focus is the utilization of system identification method to mechanize the relationship between input factors, e.g., supply voltage, armature current, SOC and the output, i.e., motor speed.

\section{Literature Review}

There are several studies with the concentration on the characteristic of battery packs. Hu, Yurkovich, and Yurkovich [1] utilized the system identification for identifying the dynamic model of the electro-thermal model of Li-ion batteries. The factors concerning this model include state-of-charge, temperature, and current direction. Juang, Kollmeyer, Jahns, and Lorenz [2] had estimated the output energy of the batteries of electric vehicles by considering the voltage and current measurement of the batteries. The system identification was also deployed by Sabatier et al. [3] in order to estimate the state of charge for the lead acid battery and the result shows that the accuracy of estimation is up to 
the error value of five percent. The system identification algorithm was developed by Sitterly, Wang, Yin, and Wang [4]. The highlight of this algorithm is the capability to self-update the identification model in the real-time model. Moreover, this study also focuses on other factors such as aging conditions and the chemical reactions of the batteries. The dynamic characteristic adaptation of the model was also discussed by $\mathrm{Hu}$, Yurkovich, and Yurkovich [5]. The proposed algorithm was tested with two types of batteries, Li-ion and NiMH and the genetic algorithm were utilized to optimize the system identification model.

Besides the linear model, the application of non-linear model was introduced by Juang et al. [6] to identify the voltage and current relationship of lead-acid batteries in the electric vehicle. The research results show that the estimation accuracy was improved significantly after the application of the new model.

\section{System Identification}

System identification method is the utilization of different statistical methods to construct a mathematical model of a dynamic system by focusing on inputs and outputs. The general equation of dynamic model is the discrete linear time-invariant, where input $(\mathrm{y}(\mathrm{t}))$ and output $(\mathrm{u}(\mathrm{t}))$ is shown as follows:

$$
y(t)=G(q) u(t)+H(q) e(t)
$$

, where $\mathrm{G}(\mathrm{q})$ is the relationship between input and output and it is represented in the form of the following transfer function or parameter of the dynamic system. The pole of the dynamic system is determined in $F(q)$.

$$
\mathrm{G}(\mathrm{q})=\mathrm{B}(\mathrm{q}) / \mathrm{F}(\mathrm{q})
$$

On the contrary, $\mathrm{H}(\mathrm{q})$ is the relationship between noise and output or parameters of noise system as shown in (3).D(q) determines the noise of the system.

$$
H(q)=C(q) / D(q)
$$

, where $\mathrm{B}(\mathrm{q}), \mathrm{F}(\mathrm{q}), \mathrm{C}(\mathrm{q})$ and $\mathrm{D}(\mathrm{q})$ are polynomials with

$$
\begin{gathered}
B(q)=b_{1} q^{-1}+\cdots+b_{n} q^{-n} \\
F(q)=f_{1} q^{-1}+\cdots+f_{n} q^{-n} \\
C(q)=c_{1} q^{-1}+\cdots+c_{n} q^{-n} \\
D(q)=d_{1} q^{-1}+\cdots+d_{n} q^{-n}
\end{gathered}
$$

, where $q-n$ is the shift operator so $q^{-n} u(t)$ is the output signal at time $\mathrm{t}=\mathrm{t}-\mathrm{n}$ or $\mathrm{u}(\mathrm{t}-\mathrm{n})$. According to (1)-(7), (8) is Box-Jenkins (BJ) model.

$$
y(t)=[B(q) / F(q)] u(t)+[C(q) / D(q)]
$$

BJ model has the capability to estimate both dynamic and noise parameters. Therefore, the construction of a model is based on the measurement noise, not the input noise. As a result, the structure of $\mathrm{BJ}$ model provides high flexibility for modeling noise. Moreover, the above model can be extended into different subclasses of how to model dynamic and noise parameters. The dynamic model can be extended into a number of models. Among these models is the output error (OE) model which focuses on the estimation of the dynamic model only while a noise model is set at the constant level and equal to 1 . The OE model is shown in (9).

$$
y(t)=[B(q) / F(q)] u(t)+e(t)
$$

These models are utilized to construct a mathematical model to explain the relationship between the output and the inputs.

\section{Battery Packs and Hybrid Vehicles}

In this study, the experiment and data collection is based on the Ni-MH batteries used in the hybrid vehicles (Figure 1 and 2). The drive shaft of a hybrid vehicle is driven by DC motor and engine (Figure 3) which are connected in parallel through the coupling. As a result, the vehicle can run on either engine or DC motor alone or both together. Moreover, not only the battery can supply voltage to DC motor but also it can act as a generator to charge the battery when it is run backward to stop the vehicle (regeneration braking).

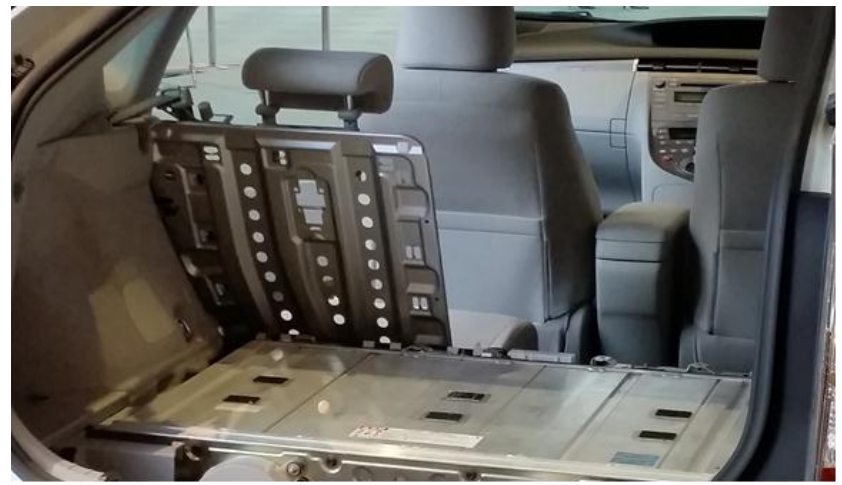

Figure 1. Ni-MH battery packs in a hybrid vehicle.

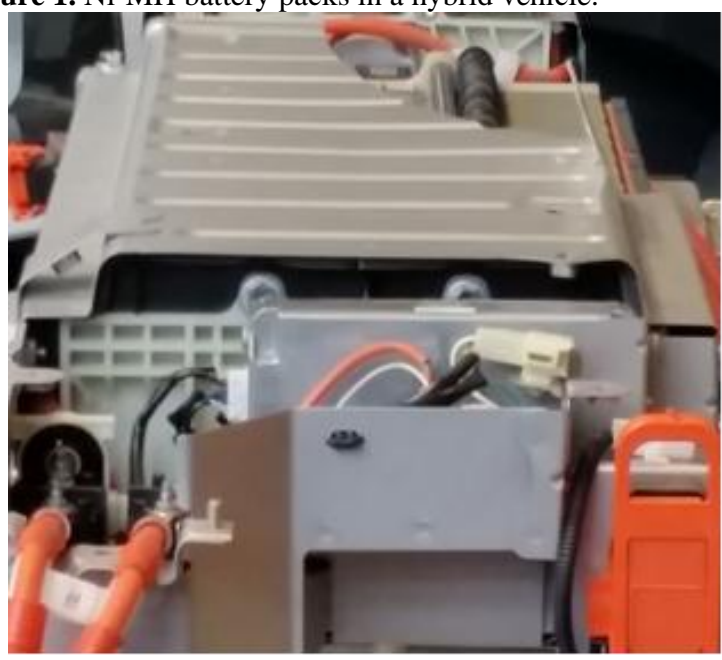

Figure 2. Ni-MH battery packs in a hybrid vehicle. 


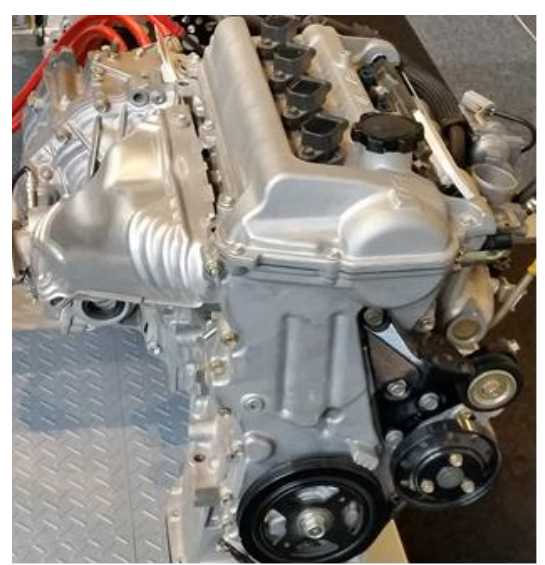

Figure 3. Engine.

\section{Data Processing}

To compare the performance of batteries for hybrid vehicles, the focused parameters are a voltage supplied from the battery, state of charge (SOC), armature current (A) and motor speed. The rated voltage of the battery is $220 \mathrm{~V}$ with the energy charge of $7 \mathrm{Ah}$. The battery is connected in parallel to a DC machine which acts as the motor and generator. The charge and the discharge of battery depend on the value of the state of charge (SOC). When the SOC us below 40 percent, a negative load torque is applied to the machine which will generate the electricity and recharge the battery. On the other hand, when SOC is above 80 percent, the load torque will be taken off so the machine will behave as a motor. All related parameters, i.e., voltage (V), SOC (percent), armature current (A) and motor speed (radian per second) will be measured and transmitted to a data logger. The make of data logger used is HOBO UX120 which has four channels.

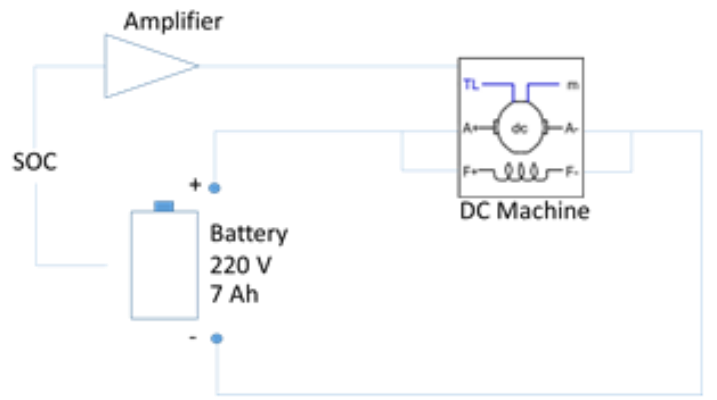

Figure 4. Schematic diagram of the battery and machine.

The real-time data of three factors, the output voltage of the battery, state of charging (SOC), and the armature current, are collected and shown in Figure 5. Due to Figure 5, the motor tends to gradually climb up when the voltage, current, and SOC increase.

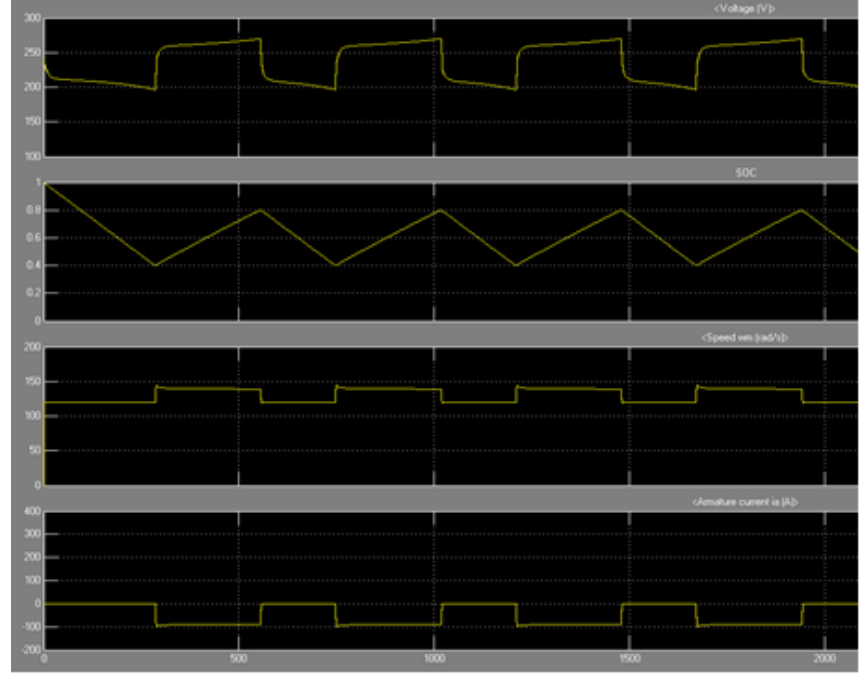

Figure 5. Outputs from Ni-MH battery and response motor speed.

\section{Model Analysis}

The system identification toolbox in MATLAB is deployed to analyze data and construct the most appropriate model to estimate the speed when the inputs are voltage, armature current, and SOC. The chosen model is the autoregressive model with exogenous input (ARX) and this result explicitly shows that both the respondent output and the input are used to derive the predicting model.

\subsection{SOC VS Speed}

The relationship between $\mathrm{SOC}$, represented by $\mathrm{u}(\mathrm{t})$ and speed, $y(t)$, is illustrated by the autoregressive model with exogenous input (ARX) which is chosen as the predicting model as follows:

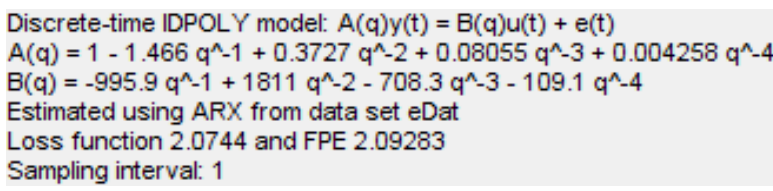

Figure 6. ARX model for SOC VS speed.

The ARX model is the combination of the utilization of the time series (historical data) or autoregressive and the external input. According to the result, the equation of $\mathrm{A}(\mathrm{q})$ and $\mathrm{B}(\mathrm{q})$ indicates that the history data of speed and SOC up to the lag of four (the highest order of lag operator is $\mathrm{q}^{-4}$ ). After the model is constructed, the simulated value of speed based on the SOC is shown in Figure 7. 

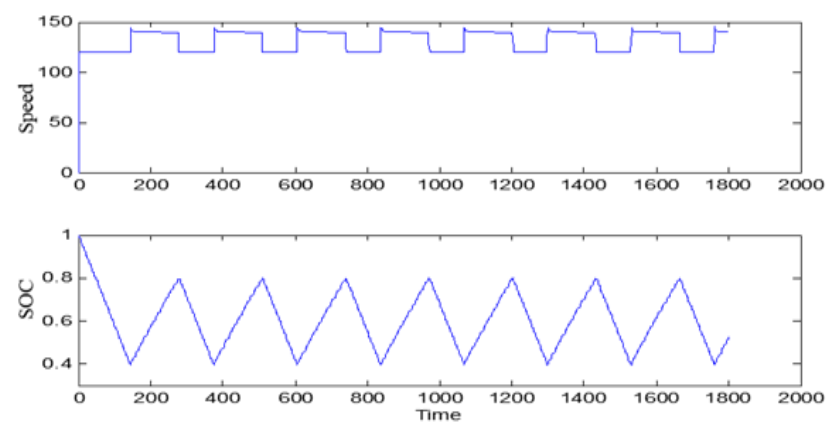

Figure 7. Simulated values of speed based on SOC.

\subsection{Voltage VS Speed}

The relationship between SOC, represented by $\mathrm{u}(\mathrm{t})$ and speed, $y(t)$, is illustrated by the autoregressive model with exogenous input (ARX) which is chosen as the predicting model as follows (Figure 8):

Discrete-time IDPOL $Y$ model: $A(q) y(t)=B(q) u(t)+e(t)$

$A(q)=1-1.466 q^{\wedge}-1+0.3727 q^{\wedge}-2+0.08055 q^{\wedge}-3+0.004258 q^{\wedge}-4$

$B(q)=-995.9 q^{\wedge}-1+1811 q^{\wedge}-2-708.3 q^{\wedge}-3-109.1 q^{\wedge}-4$

Estimated using ARX from data set eDat

Loss function 2.0744 and FPE 2.09283

Sampling interval: 1

Figure 8. ARX model for voltage VS speed

As a result, the simulated output due to the constructed model with the applied voltage is depicted in Figure 9.

\subsection{Current VS Speed}

On the other hand, the relationship between the current measured at the armature part of the motor and the speed is determined and shown in Figure 10.

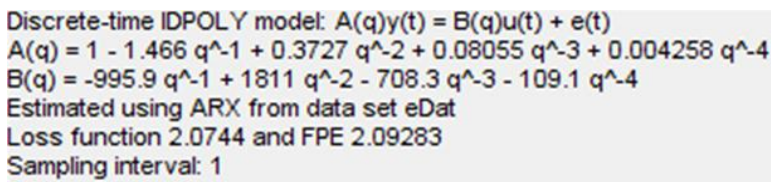

Figure 10. ARX model for armature current VS speed.

The motor speed corresponding to the armature current based on the system identification model is shown in Figure 11 .
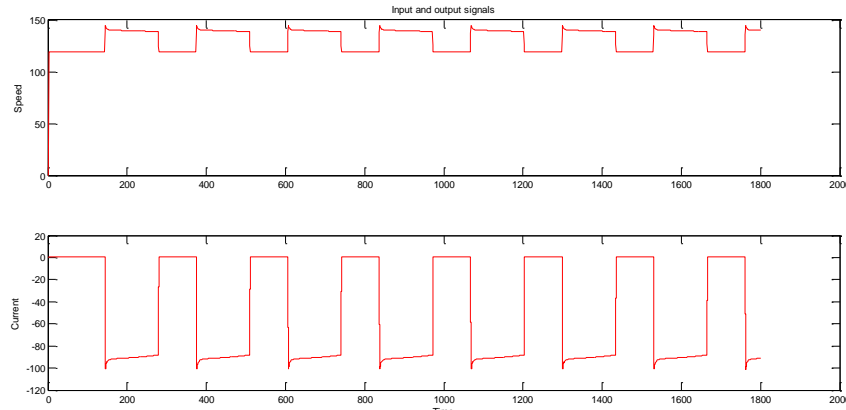

Figure 11. Simulated values of speed based on armature current.
After the system identification method is utilized in the form of ARX model to explain the relationship is these paired inputs and outputs: SOC VS Speed, Voltage VS speed and Current VS speed, the results show that the output speed can be efficiently predicted by a single input (SOC, voltage and current) and their historical data. The difference between the actual value of the motor speed based on the system identification model and the actual value is shown in Table 1. According to the results in Table 1, there is no significant difference between the actual speed and the predicting value based on the ARX model. According to the results in Table 1, there is no significant difference between the actual speed and the predicting value based on the ARX model since the difference is ranged from 1.05 to only 2.36 percent. The lowest is from the prediction of SOC by ARX model (1.05 percent) while the highest is the voltage prediction $(2.36$ percent)

Table 1. Difference between actual and predicting values.

\begin{tabular}{|c|c|}
\hline Input of ARX & $\begin{array}{c}\text { \% Difference between actual speed and } \\
\text { predicting value }\end{array}$ \\
\hline SOC & 1.05 \\
\hline Voltage & 2.36 \\
\hline current & 1.42 \\
\hline
\end{tabular}

\section{Conclusions and Discussions}

The characteristics of the Ni-MH battery pack in hybrid vehicles, i.e., voltage, current and SOC, and the motor speed are studied to determine the relationship between these inputs and the output speed. All data is measured and transmitted to the data logger. Afterwards, the system identification method is utilized to characterize the statistical model explaining the relationship. The chosen model is the autoregressive model with exogenous input (ARX). After comparing the actual and simulated scenario, the result indicates that the constructed model can simulate the output speed accurately. The results show that the empirical model can be utilized without the need to derive the mathematical model between the input and the output. Therefore, the proposed model is capable to characterize the performance of the motor of hybrid vehicles when there are different inputs and their values are varied.

\section{References}

1. Y. Hu, S. Yurkovich, and B.J. Yurkovich, J. Power Sources 196, 449-457 (2011).

2. L. W. Juang, P. J. Kollmeyer, T. M. Jahns, and R. D. Lorenz, Proc. IEEE Energy Conversion Congress and Exposition (ECCE) 3903-3910 (2010).

3. J. Sabatier, M. Aoun, A. Oustaloup, G. Gregoire, F. Ragot, and P. Roy, Signal Process., 86, 26452657(2006).

4. M. Sitterly, L. Y. Wang, G. G. Yin, and C. Wang, IEEE Trans. Sustain. Energy 2, 300-308(2011).

5. Y. Hu, S. Yurkovich, and B.J. Yurkovich, Control Eng. Pract. 17, 1190-1201(2009). 
6. L. W. Juang, P. J. Kollmeyer, T. M. Jahns, and R. D. Lorenz, IEEE Trans. Ind. Appl. 49, 1480-1483(2013).

7. O. Tremblay, L. -A. Dessaint, A. -I. Dekkiche, proc. IEEE Vehicle Power and Propulsion Conference (2007). 